\title{
INVESTIGACIÓN
}

Recibido: 12/03/2021 --- Aceptado: 15/04/2021 --- Publicado: 24/05/2021

\section{EL USO DE LAS REDES SOCIALES Y SU RELACIÓN CON LA DECISIÓN DE COMPRA DEL TURISTA}

\section{The use of social media and its relation to the tourist's purchase decision}

David Caldevilla-Domínguez: Universidad Complutense de Madrid. UCM. España davidcaldevilla@ccinf.ucm.es

Almudena Barrientos-Báez: Universidad Europea de Madrid. UEM. España almudena.barrientos@universidadeuropea.es

Álvaro Pérez-García: Universidad Internacional de La Rioja. UNIR. España alvaro.perezgarcia@unir.net

M. Gloria Gallego-Jiménez: Universidad Internacional de La Rioja. UNIR. España gloria.gallego@unir.net

Cómo citar el artículo:

Caldevilla-Domínguez, D., Barrientos-Báez, A., Pérez-García, A., y Gallego-Jiménez, M. G. (2021). El uso de las redes sociales y su relación con la decisión de compra del turista. Vivat Academia. Revista de Comunicación, 154, 443-458. http://doi.org/10.15178/va.2021.154.e1360

\section{RESUMEN}

Tras la depresión provocada a escala internacional por la pandemia de la covid-19, el sector del turismo necesita recuperarse en un contexto de falta de capital para inversiones. En ese sentido, el contacto rápido y económico con el cliente mediante medios digitales se va a volver si cabe, más importante, haciendo referencia a la facilidad de acceso, búsqueda y selección de ofertas y simplificación de trámites económicos. El comportamiento del viajero ha cambiado sustancialmente a raíz de la digitalización de la sociedad, que ha mejorado exponencialmente el uso de las TIC en los últimos meses de 2020. Aumenta así, la necesidad de investigar y acudir a estudios que nos permiten entender el nuevo ecosistema en el que empresas y compradores se relacionan. Este trabajo consiste en una revisión bibliográfica orientada al rastreo y recuperación de información relevante a la aplicación de las redes sociales en la promoción de la industria turística por sus efectos en la relación empresa-cliente. Para el caso, se han consultado diversas fuentes en materias 
Caldevilla-Domínguez, D., Barrientos-Báez, A., Pérez-García, A. y Gallego-Jiménez, M. G. El uso de las redes sociales y su relación con la decisión de compra del turista

relacionadas y transversales: monografías, artículos, y revistas científicas de publicación reciente en español e inglés y con acceso abierto (open access) al texto completo (incluyendo material más antiguo solo si contiene una base teórica relevante). Los criterios de exclusión incluyeron artículos faltantes a la rigurosidad científica, de opinión, o contenido de usuario sin base en experiencia. La estrategia de búsqueda se sirvió de Descriptores de turismo, comunicación y TIC relevantes para el objeto de estudio.

PALABRAS ClAVE: Comunicación - Consumidor - Covid-19 - Redes sociales Relaciones públicas - TIC - Turismo.

\section{ABSTRACT:}

Following the international depression caused by the covid-19 pandemic, the tourism sector needs to recover in a context of lack of capital for investment. In this sense, fast and economical contact with the customer through digital means is going to become, if possible, more important, referring to the ease of access, search and selection of offers and simplification of financial procedures. Traveler behavior has changed substantially as a result of the digitalization of society, which has exponentially improved the use of ICT in the last months of 2020. Thus, the need to research and go to studies that allow us to understand the new ecosystem increases in which companies and buyers are related. This work consists of a bibliographic review aimed at tracking and recovering information relevant to the application of social networks in promoting the tourism industry due to its effects on the company-client relationship. For this case, various sources have been consulted in related and crosscutting subjects: monographs, articles, and scientific journals of recent publication in Spanish and English and with open access to the full text (including older material only if it contains a base relevant theory). Exclusion criteria included articles lacking scientific rigor, opinion, or user content with no basis on experience. The search strategy used descriptors of tourism, communication and ICT relevant to the object of study.

KEYWORDS: Communication - Consumer - Covid-19 - Public relations - Social networks - TIC - Tourism.

\section{INTRODUCCIÓN}

Es un signo de nuestros días el de la lectura y escritura pantalla y teclado frente al tradicional medio caligráfico, una tendencia que ha llevado a que, en esta era digital el lenguaje y sus manifestaciones varíen significativamente respecto a patrones pasados, llegando al extremo de no poder identificar correctamente si la comunicación se está produciendo de manera escrita o hablada en entornos de carácter híbrido (chat, mensajería móvil, redes sociales, etc.). El auge de los medios audiovisuales puede invitar a concebir una realidad no necesitada de habilidad escritora, pero ésta es socialmente imprescindible a la par de las destrezas orales. Potenciadas a su manera por nuevos tipos de literacidad (la digital, concretamente) y 
Caldevilla-Domínguez, D., Barrientos-Báez, A., Pérez-García, A. y Gallego-Jiménez, M. G. El uso de las redes sociales y su relación con la decisión de compra del turista

nuevos géneros híbridos y multimodales con sus propios rasgos verbales, visuales o auditivos.

En este contexto de medios y literatura digital, se cruzan dos ámbitos que apriorísticamente pueden tener un carácter opuesto (Ganga-Contreras et al., 2017): la cultura participativa de prosumidores promovida e incluso impuesta por el entorno digital, y la industria: la turística, en nuestro caso. En la primera existen prácticas como la remezcla, la inteligencia colectiva y la cultura de colaborativa entre prosumidores, aficionados y coinasseurs, mientras que el segundo practica la homogeneización de los procesos productivos, la búsqueda de lucro y la concentración empresarial. Entre ambos existe una zona gris. Es aquí donde usuarios con competencias digitales intermedias están influenciando las industrias turísticas, en concreto, en la prescripción de productos o servicios mediante redes sociales (Matassi y Boczkowski, 2020; Araújo y González-Valiente, 2019).

Este trabajo surge de las reflexiones sobre este nuevo ecosistema mediático y el papel desempeñado por los usuarios en el mismo, así como en los retos y problemáticas que se originan en las relaciones entre prosumidores y la industria turística.

En el sector turístico, las redes sociales están cambiando el paradigma de la comunicación, cambiando la forma en que las personas acceden a la información, planifican y comparten sus viajes. Por otro lado, permiten a las empresas interactuar de forma dinámica y directa con el usuario final, permitiendo la adaptación de su actividad a las nuevas realidades, la evaluación del nivel de satisfacción, la implantación de mejoras y la difusión de su oferta. En este contexto, Instagram se destaca como la red social que permite estimular el diálogo a través del intercambio de fotografías, vídeos o experiencias sobre un determinado destino o producto turístico.

Sin embargo, varios estudios muestran que todavía se acusa una falta de conocimiento sobre cómo las iniciativas rurales pueden utilizar las redes sociales de manera eficiente y efectiva (Oliveira et al., 2017; Hays et al., 2013; Infante Limón, 2014). Estas redes sociales ganan fuerza a partir de ambientes específicos, entre ellos el Instagram (Calderón et al., 2017), que posee capacidad de transferir mensajes a partir de la imagen.

Este artículo pretende contribuir a una mayor comprensión del potencial de las redes sociales.

\section{OBJETIVOS}

Conocer el ecosistema en el que empresas y compradores se relacionan a través del uso de las TIC en la industria turística. 
Caldevilla-Domínguez, D., Barrientos-Báez, A., Pérez-García, A. y Gallego-Jiménez, M. G. El uso de las redes sociales y su relación con la decisión de compra del turista

\section{METODOLOGÍA}

Este trabajo consiste en una revisión narrativa orientada al rastreo y recuperación de información relevante a la aplicación de las TIC en la promoción de hábitos saludables por sus beneficios físicos pero también emocionales, dando respuesta al planteamiento inicial. Para el caso, se han consultado diversas fuentes en materia de TIC, comunicación y Ciencias de la Salud, en libros, artículos, estudios y revistas científicas. Los criterios de búsqueda de información incluyeron: estudios, artículos, monografías, etc., publicados entre 2010-2020 en español o inglés y con acceso abierto $\mathrm{u}$ open access al texto completo (con la posibilidad de dejar paso a material más antiguo si sienta una base teórica relevante). Los criterios de exclusión incluyeron artículos de carácter marcadamente contrario al científico, como artículos de opinión, o material de acceso informático producido por el usuario sin base en experticia. La estrategia de búsqueda se sirvió de Descriptores de Ciencias de la Salud (DeCS), comunicación y TIC relevantes para el objeto de estudio. La influencia de las nuevas tecnologías en relación a la comunicación es cada vez mayor, pues ofrecen posibilidades que hasta hace poco, eran impensables (Barrientos-Báez, 2016).

\section{DISCUSIÓN}

Como tal fenómeno, el turismo puede decirse que tiene elementos $y$ manifestaciones que predatan a su popularización como industria y sector económico propio, y sin embargo ha producido ramas de negocio muy asequibles a la publicidad asimétrica, como el turismo de motivación cultural (Araújo y Domínguez, 2012). El mercado por sí mismo ha estado generando estas ramas y aprovechando cada nueva forma de comunicación sobre la base popular del modelo "sol y playa" respondiendo así a una variedad de audiencias, segmentadas para ampliar las oportunidades comerciales (Caldevilla-Domínguez et al., 2019). Ello ha aumentado la competitividad del sector al haber diversificado la oferta, tanto en términos de productos principales, como de los secundarios o complementarios (Bonet, 2003). Como delatan los hechos recientes, la apuesta por el sector, pese a todo, no es en sí misma garantía de una base económica propiciadora de desarrollo (Fiorello, 2010; Boccardi et al., 2008), pero constituye indudablemente un activo capital de la economía, en particular a nivel local, por su capacidad de crear empleos que permitan complementar ingresos públicos y privados pese a su estacionalidad.

No es superfluo hacer mención a los efectos perniciosos de la mercantilización de entornos naturales o bienes culturales, responsable potencial de su adulteración, de daños al entorno natural (Celso Campos, 2010), y de desvirtuación de sus fines humanos originales (Richards, 2004; de Sena Abrahão y Tomazzoni, 2017) aplicables también a la digitalización de las experiencias (Caldevilla-Domínguez et al., 2020).

Siguiendo a César-Arnaiz y Arnaiz-Burne (2016):

El turismo ha dejado de ser la expresión para formar parte de las reglas nuevas del desarrollo mundial. Ayer fueron pocos los que podían viajar y 
Caldevilla-Domínguez, D., Barrientos-Báez, A., Pérez-García, A. y Gallego-Jiménez, M. G. El uso de las redes sociales y su relación con la decisión de compra del turista

disfrutar de un sistema de vida que era de las minorías, hoy el turismo ha socializado el ocio, pero detrás de esto hay algo más.

Los procesos de recuperación de las grandes explotaciones coloniales los realiza el turismo que puede transformar los establecimientos de la esclavitud en "paraísos", dando lugar al nacimiento de la denominada cuarta plantación, el monocultivo del turismo, que domina el Caribe y muchas islas del Pacífico.

El fin de la sociedad del trabajo y el nacimiento de una nueva donde no se saben los límites del trabajo y el ocio, no podría existir sin el turismo, que puede disfrazar la explotación como un viaje de premio a la productividad o permite combinar el sol con el trabajo en el ordenador, manteniendo siempre la percepción al sujeto que es "libre", para auto explotarse o dejarse caer en el "abandono" una muerte civil por falta de dinero. El turismo recicla las historias, redefine los territorios y logra mover grandes cantidades de personas a sus nuevos destinos, que a medida que van "envejeciendo" los van dejando por otros nuevos y éstos son ocupados por la masividad.

La relación sistema-sociedad y turismo está unida por tres grandes instituciones: el mercado que es el punto de encuentro e intercambio, los imaginarios que son las guías del deseo y el dinero electrónico que es el pasaporte a la felicidad: ese espacio que antes se llamaba vacaciones y hoy simplemente es turismo.

El turismo es probablemente una de las actividades que han recibido mayor influencia de las Tecnologías de la Información y de la Comunicación -TIC(Altamirano et al., 2016) respecto a su formulación previa (Correyero, 2003) ya que las Relaciones públicas de las que se sirven para su popularización beben de estas fuentes (Caldevilla, 2007) y eso implica también los problemas tocantes a bulos y desinformación (Barrientos-Báez et al., 2018).

Los avances en comunicación digital, han promovido modificaciones notables y profundas tanto en la promoción como en la difusión (Alves et al., 2016), así como en lo relativo a los planes y a la efectiva práctica comercial del sector. La acción de las nuevas tecnologías de la comunicación digital invade la propia experiencia del viaje gracias a la telepantalla moderna: los dispositivos móviles con aplicaciones de geolocalización capaces de asistir al viajero en términos vitales y accesorios. Las redes sociales afectan a la percepción publicitaria, al facilitar el contraste de la experiencia anunciada con la impresión de miles de viajeros.

El turista moderno ya no es ese viajero al que se ha llegado a comparar con rebaño in-contestatario de las agencias: es el turista 3.0. Un aventurero independiente que modifica el esquema "vertical" de viaje: "más informado que nunca, participativo, que busca y compara y que ya no decide a partir del consejo de una agencia de viajes, sino que ha de ser persuadido directamente por el producto o servicio turístico y la forma en que éste se presenta en Internet" (Suau, 2012, p. 145); lo que lo torna en un prosumidor con funciones de anunciante/publicista: crítico, si se prefiere, del contenido que consume. Citando el célebre slogan "busca, compara y si encuentra algo mejor...". Y no solo eso, sino que comunica sus impresiones mediante redes 
Caldevilla-Domínguez, D., Barrientos-Báez, A., Pérez-García, A. y Gallego-Jiménez, M. G. El uso de las redes sociales y su relación con la decisión de compra del turista

sociales y centros de mensajería carentes de filtro o control de la industria, generando contenido crítico y afectando al capital reputacional en destino (Caro et al., 2014). Todo ello convierte al turista 2.0 es un promotor turístico independiente y tecnológicamente competente, que se apoya en una comunidad digital colaborativa (Sarmiento y Rodríguez, 2018).

Este nuevo turista elige y compra sus productos del ramo de manera distinta:

“En el caso del turismo, el proceso se inicia con el surgimiento de la necesidad de viajar, por los motivos que sean. El consumidor buscará, a continuación, información sobre las ofertas existentes en el mercado que puedan satisfacer de forma adecuada su necesidad. La fase posterior consistirá en la evaluación de las distintas alternativas que se le ofrecen. Después del proceso de evaluación de las alternativas, el consumidor seleccionará la que considere satisface de forma más conveniente su necesidad... Por último, una vez utilizado el servicio, se entra en la última fase, y no por ello menos importante, sino todo lo contrario, de evaluación del grado de satisfacción con la compra efectuada... Todo el proceso de decisión de compra está influido y condicionado, tanto por variables internas del individuo como por variables externas, además de los estímulos de marketing a los que puede verse expuesto el consumidor" (Serra, 2011).

Ortega (2012), afirma que el proceso de decisión de compra, en el caso del viajero 2.0, no termina con la compra y contratación: prosigue en destino durante el consumo del producto, que implicará a su vez, la contratación de servicios: museos, visitas, comidas, etc., que generarán experiencias propias y afectarán a la existente. Llamadas académicamente "decisiones secundarias", por ser accesorias respecto a las decisiones principales o que se toman en los mercados primarios de donde generalmente provienen estos turistas de segunda generación.

Según Altamirano Benítez et al. (2016) este turista, como internauta que es, no se desconecta durante viajes de ocio o turismo: Internet continúa siendo una herramienta vital primaria cuando llegan a destino. Al fin y al cabo, la red está estrechamente relacionada con variadas formas de consumo (Gonzálvez et al., 2020). Según los datos citados por Benítez, el $81 \%$ la utiliza para informarse sobre qué lugares visitar. Alrededor de dos tercios para tomar decisiones sobre la oferta de ocio y cultural y hotel (69\% y 63\% respectivamente). Destaca también el uso de las apps GPS (Google Maps) ya entonces en manos de un cuarto de los internautas (29\%). Altamirano también cita datos sobre el papel de anunciantes/promotores de los turistas 2.0: el $49 \%$ ha accedido a la Internet para compartir experiencias positivas en lo tocante al servicio recibido en establecimientos o desplazamientos, mientras que un $35 \%$ comparte en redes experiencias negativas.

Este turista 2.0 y su nuevo set de necesidades y capacidades han creado sus propias comunidades online con influencia en la selección de productos del sector turístico. Surgen de forma autónoma y generalmente, independientemente de la industria, como ya se ha señalado. Todo ello tiene un inevitable efecto en la 
Caldevilla-Domínguez, D., Barrientos-Báez, A., Pérez-García, A. y Gallego-Jiménez, M. G. El uso de las redes sociales y su relación con la decisión de compra del turista

comunicación con sus públicos de las instituciones y compañías del sector, obligando a repensar los planes de comunicación.

Acorde a Domínguez y Araújo (2012; 2014) la clave en una comunicación digital eficaz no reside solo en contar con presencia en dicho entorno digital, desde la que generar información para el turista: sino en "la búsqueda de modos de gestionarla y hacerla atractiva para los usuarios" orientada a crear la necesidad de consumir el producto. Esto es: de realizar el viaje.

El turista 2.0, con su competencia digital (Fernández-Cavia et al., 2020), puede acceder directamente a la oferta saltándose los intermediarios con sus compras online. Enfrentadas al omnipresente fenómeno de la infoxicación, las empresas deben recurrir al valor añadido para seguir siendo relevantes al cliente, mediante contenidos de calidad y herramientas útiles en sus portales y redes sociales.

Hoy por hoy toda estrategia de comunicación comercial ha de ir complementada o incluso dirigida por la creación de cuentas en redes sociales (Navarro et al., 2020; Chinchay-Villarreyes et al., 2020) especializadas y generalistas, apuntadas al establecimiento de una comunicación interactiva en la que el usuario participe motu proprio (Martín-Antoranz et al., 2019) creando contenidos y promoviendo los destinos turísticos. Las plataformas digitales tienen, en fin, la capacidad de promocionar la esta oferta, pero por la misma vertiente el turista 2.0 exige experiencias y contenidos trabajados que le inspiren a consumir y apoyar el producto.

\section{RESULTADOS}

Entendiendo las redes sociales en relación al turismo como aquellas en las que los usuarios comparten sus experiencias vacacionales y opiniones, podemos apuntar como representativas a las redes Facebook, Twitter e Instagram. A principios de 2019, el número de usuarios de red social alcanzaba los 3.500 millones: casi la mitad de los 7000 millones de seres humanos en la tierra, [Digital in 2019 | Global Digital Report de We Are Social (2019)]. De esos 3.500 millones, 3.256 se conectan a las mismas, total o parcialmente a través de dispositivos móviles, que han dado el gran paso de convertirse en el principal dispositivo digital de la mayoría de usuarios.

De entre las redes mencionadas, Facebook continúa siendo líder mundial en número de usuarios, con aproximadamente 2.271 millones en todas las plataformas (PC's de sobremesa, portátiles, tabletas, y como ya se ha mencionado: smartphones un $96 \%$ de esos 2.000 millones-). Estando destinada a compartir intereses de todo tipo: tanto personales como profesionales de los usuarios, es asimismo la principal red social en términos de contenido turístico. Facebook permite y supone una variedad enorme de contenido, lo que unido a sus herramientas de seguimiento y análisis, la convierte en una red social de gran utilidad en la gestión de redes sociales para el sector turístico. 
Caldevilla-Domínguez, D., Barrientos-Báez, A., Pérez-García, A. y Gallego-Jiménez, M. G. El uso de las redes sociales y su relación con la decisión de compra del turista

Otra de las redes sociales de mayor relevancia es Twitter: el hogar de las polémicas. Aunque en 2019 su actividad descendido un 2,7\% respecto al pasado año (We Are Social, 2019), sus 326 millones de usuarios continúan nutriendo de contenidos esta plataforma asiduamente, atraídos por la posibilidad de la mensajería breve y rápida y por su encendida polémica. Esta red social apunta a la instantaneidad de la comunicación con los seguidores y a la publicidad asociada a aparecer en las tendencias de "hashtags" y que permite llegar a audiencias normalmente no al alcance del marketing convencional.

Finalmente, la plataforma en auge y con mayores prospectivas de futuro parece ser Instagram: con más de 1.000 millones de seguidores, pero sin embargo propiedad de Facebook desde 2012. Su crecimiento no se ha detenido en este tiempo, y a este ritmo podría alcanzar las cifras de Facebook convirtiéndose en su propia competencia dentro del mismo grupo (Lalueza, 2019). Sin que por ello el nivel de engagement en Instagram parezca ser un problema insuperable para la flexibilidad de contenidos de Facebook.

Cada plataforma tiene sus ventajas e inconvenientes, dependiendo, primeramente, de las tendencias de sus seguidores en cuanto a consumo y formas de consumo; y siguiendo por los del público objetivo y sus redes de preferencia.

En el contexto publicitario, la red es un lugar de encuentro entre empresas y usuarios en donde la comunicación funcional debe organizarse de manera horizontal: entre iguales, a fin de crear un firme vínculo directo y de confianza con el público objetivo, que genere el deseo de interactuar con la empresa, pero también el de compartir contenido con la red de contactos (Sánchez-Torres y Restrepo, 2015). Se busca generar engagement "que es algo más complejo que generar un me gusta o retweets [...] Los mercadólogos deben tener claras las percepciones de sus consumidores, sus deseos, necesidades y preferencias" (2015, p. 45).

Para conectar de esta manera con el prosumidor, las empresas están condenadas a un "ritmo vertiginoso" paralelo al que el público desarrolla por la red "las decisiones se toman en segundos, porque cada vez los usuarios están más conectados al mundo, cuentan con un mayor acceso a la información y no solo son consumidores de contenido sino [...] prosumidores hiperconectados e hiperinformados" (SánchezTorres y Restrepo, 2015, p. 34). Por ello una de las estrategias más importantes en redes sociales es reaccionar en tiempo real (real time marketing). En 2015, el estudio Real Time Marketing "Report de Monetate y Econsultancy" determinó que un 70\% de las empresas adolecía de lentitud en sus actuaciones de marketing y lo reconocían. Un factor que en el presente contexto resulta indispensable para incrementar la presencia en redes, con los efectos que ello tiene en la fidelización y captación de prosumidores de cualquier producto. Sin espacio para la improvisación, las empresas deben estudiar el comportamiento en las redes sociales de sus públicos a fin de desarrollar la capacidad de ajustar su comunicación, atención y comercialización a las exigencias de cada circunstancia. 
Caldevilla-Domínguez, D., Barrientos-Báez, A., Pérez-García, A. y Gallego-Jiménez, M. G. El uso de las redes sociales y su relación con la decisión de compra del turista

La cantidad de tendencias que surgen diariamente de las redes sociales y de las que las marcas podrían aprovecharse son incontables; pero deben ser evaluadas profesionalmente para determinar cuáles conciernen a su audiencia y nicho de público concreto (Sánchez-Torres y Restrepo, 2015, p. 43).

Las redes sociales constituyen un espacio ideal para investigar el mercado y conocer las particularidades de los usuarios, sobre todo de los nativos digitales mayores de edad, entre 18 y 30 años. "Conocer la personalidad de los demás es una habilidad esencial para tener relaciones sociales exitosas" (Martínez-Valerio, 2016, p. 149) y en este contexto de comunicación digital, las redes sociales se erigen como una plataforma en una puerta abierta al interior de la mente de estas generaciones.

Desde que Facebook comenzó su andadura en el año 2006, ha despertado un remarcable interés entre la comunidad científica por su capacidad para exponer la personalidad de sus usuarios (Stutzman, 2006; Grasmuck et al., 2008; Mayer y Puller, 2008; Pempek et al., 2009; Back et al., 2010; Goldbeck, 2011; Junco, 2011), creándose numerosos trabajos en torno al contexto académico, universitario y de consumo.

Si bien este interés ha venido acompañado de cierta discrepancia respecto a la veracidad de la identidad mostrada por los usuarios en estos entornos online, Cáceres et al., 2009 y Chen y Marcus, 2012, coinciden en que se crea una cierta deformación de la personalidad "real" en el entorno digital), la mayoría de investigadores coinciden en que los principales rasgos identificativos que una persona publica en Facebook se corresponden con los de su vida real (Back et al., 2010; Gosling et al., 2011). En este sentido, los resultados de una las más grandes investigaciones llevadas a cabo sobre Facebook (Kosinski, 2013) apuntan a la existencia de una relación entre la personalidad, el perfil en la red social y las visitas a determinados sitios web. Para dicho estudio, los autores diseñaron la aplicación "My Personality" obteniendo datos sobre la personalidad de casi 7 millones y medio de usuarios sobre la base de sus "Me gusta" (Parra et al., 2014).

Investigaciones más recientes señalan todavía en esta dirección en lo relativo a la honestidad de los usuarios en redes sociales, demostrando que "es posible conocer mejor a una persona incluso mejor que su propia familia a través de [...] redes sociales" (Youyou et al., 2015, citado en Martínez-Valerio, 2016, p. 152).

\section{CONCLUSIONES}

El turista ha pasado a convertirse en un sujeto activo del proceso en la industria turística: más allá de su importancia previa como cliente, a día de hoy tiene un papel como anunciante, promotor y crítico de los productos que consume y de los servicios que se le ofrecen. Según (Salazar et al., 2020) los propios actores, desde sus prácticas comunicativas, imponen una permanente tensión a la crítica, definiendo los límites y la legitimidad de su ejercicio, según el contexto y las particularidades de la temática objeto de atención. 
Caldevilla-Domínguez, D., Barrientos-Báez, A., Pérez-García, A. y Gallego-Jiménez, M. G. El uso de las redes sociales y su relación con la decisión de compra del turista

Esto supone un cambio de paradigma considerable con respecto a su papel tradicional, y plantea la necesidad de replantear las estrategias de comunicación sobre la base de planteamientos propios del entorno digital del que los nuevos públicos del sector turístico son nativos: un entorno en el que existen comunidades colaborativas propias. Comunidades dedicadas y voluntariamente apasionadas en el comentario independiente de los productos turísticos, con las consecuencias que ello puede tener al quitar buena parte del control reputacional de las manos de las empresas.

Estas estrategias pasan por servirse de las redes sociales para generar una interactividad constante en la relación con el cliente, de manera que este se sienta constante y adecuadamente atendido en sus necesidades. Esta comunicación facilita alas a la compra de productos turísticos y a su administración mediante medios informáticos, eliminando intermediarios, reduciendo en buena medida los costes y dando al viajero un conocimiento sin precedentes sobre el sector y la oferta. Esta tendencia inevitable del comercio hacia el 2.0 implica necesariamente una ausencia de suficiente base teórica para cubrir todos los aspectos relevantes, que permita establecer los parámetros fundamentales de lo que será del marketing y la publicidad turística en un futuro cercano. Reflejo de esto es el surgimiento de comunidades virtuales de forma casi exclusivamente voluntaria: sin apenas intervención de la industria.

También obliga a vigilar los aspectos secundarios de la experiencia del viajero, a fin de impedir que una mala experiencia menor, expuesta por un tercero, pueda tener consecuencias negativas en la impresión de un gran número de usuarios.

La estrategia más conveniente se halla en la diferenciación mediante contenidos de calidad, y en la atención a las experiencias secundarias que el cliente consume una vez inicia el viaje: atendiendo así, tanto a la satisfacción del cliente como a la necesidad de presencia online y al posicionamiento en buscadores, clave desde el punto de vista de las TIC. El sector turístico ha hecho uso de la web 2.0 y en especial de las redes sociales, convirtiéndolas en herramientas promocionales del día a día, haciendo que surjan redes sociales específicas: comunidades virtuales iniciadas por los usuarios. Para cualquier institución o compañía turística es imprescindible saber gestionarse en el entorno de las redes sociales para relacionarse con sus usuarios o viajeros en potencia. La opinión que estos usuarios compartan posteriormente, ayudará a otros usuarios a la hora de tomar decisiones de compra y de contratación de servicios.

De igual modo, la imagen online de destino turístico es un factor determinante en la decisión del turista, ya que constituye la principal fuente de información reputacional para él. A día de hoy múltiples redes sociales están a disposición del turista para recurrir a ellas a la hora de organizar un viaje: desde las que sólo divulgan información de destinos turísticos, alojamientos o centros de visitantes, hasta las construidas en torno a una interacción más amplia entre el usuario 2.0 y la plataforma. Según Barrientos-Báez et al. (2017) las TIC y sus aplicaciones, pueden 
Caldevilla-Domínguez, D., Barrientos-Báez, A., Pérez-García, A. y Gallego-Jiménez, M. G. El uso de las redes sociales y su relación con la decisión de compra del turista

clasificarse con facilidad en función de su finalidad y servicio que ofertan, y se distinguen por estar enfocadas hacia un público objetivo y sus necesidades.

Al hablar de internet, web 2.0 o más concretamente de redes sociales y el papel de la interactividad en este nuevo estado de cosas, se abren una serie de oportunidades para el sector, en lo relativo a establecer vínculos con los viajeros y a controlar interactivamente su impresión reputacional del servicio que disfruta.

\section{REFERENCIAS}

Abrahão, C. M. y Tomazzoni, E. (2017). Turismo de Segundas Residências: análise dos conflitos territoriais no destino de Matinhos (Litoral do Paraná, Brasil). Fronteiras: Journal of Social, Technological and Environmental Science, 6(1), 143-162. https://doi.org/10.21664/2238-8869.2017v6i1.p143-162

Altamirano Benítez, V. P., Túñez López, J. M. y Valarezo González, K. P. (2016). Promoción turística 2.0. Análisis de las redes sociales de los gobiernos iberoamericanos. Opción, 32(Especial 9), 32-53. https://cutt.ly/7jq1CqZ

Alves, L., Antunes, N., Agrici, O., Sousa, C. y Ramos, C. (2016). Click Bait: You Won't Believe What Happens Next!. Fronteiras: Journal of Social, Technological and Environmental Science, 5(2), 196-213. https://doi.org/10.21664/22388869.2016v5i2.p196-213

Araújo Vila, N. y Domínguez Vila, T. (2012). Los festivales de cine como elemento potenciador de destinos turísticos. El caso de San Sebastián. Revista de Comunicación Vivat Academia, 31-49. http://dx.doi.org/10.15178/va.2012.121.31-49

Araújo Vila, N. y Domínguez Vila, T. (2014). Gestión de las Redes sociales turísticas en la web 2.0. Revista de Comunicación Vivat Academia, 129, 57-78. https://doi.org/10.15178/va.2014.129.57-78

Araújo, C. A. y González-Valiente, C. L. (2019). Hacia un pensamiento informacional iberoamericano. Bibliotecas. Anales de Investigación, 15(2), 137-139. https://cutt.ly/Djq0uh3

Arcila Calderón, C., López, M. y Pena, J. (2017). El efecto condicional indirecto de la expectativa de rendimiento en el uso de Facebook, Google+, Instagram y Twitter por jóvenes. Revista Latina de Comunicación Social, 72, 590-607. http://doi.org/10.4185/RLCS-2017-1181

Back, M., Stopfer, J., Vazire, S., Gaddis, S., Schmukle, S., Egloff, B. y Gosling, S. (2010). Facebook Profiles Reflect Actual Personality, Not Self-Idealization. Psychological Science, 21(3), 372-374. http://doi.org/10.1177/0956797609360756 
Caldevilla-Domínguez, D., Barrientos-Báez, A., Pérez-García, A. y Gallego-Jiménez, M. G. El uso de las redes sociales y su relación con la decisión de compra del turista

Barrientos-Báez, A. (2016). GDS Amadeus. Propuesta de innovación didáctica. En TIC actualizadas para una nueva docencia universitaria. McGraw Hill.

Barrientos-Báez, A., Barquero Cabrero, M. y García García, E. (2018). Posverdad y comunicación 2.0: el reto periodístico de una era sin periodistas. Revista de Ciencias de la Comunicación e Información, 23(1), 43-52. http://doi.org/10.35742/rcci.2018.23(1).43-52

Barrientos-Báez, A., Caldevilla-Domínguez, D. y García-García, E. (2017). APP para la tercera edad: utilidad, clases y valor social. Revista de Ciencias de la Comunicación e Información, 22(2), 111. http://doi.org/10.35742/rcci.2017.22(2).1-11

Boccardi, F., Orellana, M., Giovannini, S. y Rocchietti, D. (2008). El sueño minero: un análisis de la narrativa utópica del desarrollo. Perspectivas de la Comunicación, 1(1), 56-72. http://revistas.ufro.cl/ojs/index.php/perspectivas/article/view/10

Bonet, L. (2003) Turismo Cultural: Una reflexión desde la ciencia económica. Portal iberoamericano de Gestión Cultural. https://cutt.ly/Ojq0mId

Cáceres, M. D., Ruiz-San-Román, J. A. y Brändler, G. (2009). Comunicación interpersonal y vida cotidiana. La presentación de la identidad de los jóvenes en Internet. CIC. Cuadernos de Información y Comunicación, 14, 213-231. https://revistas.ucm.es/index.php/CIYC/article/view/CIYC0909110213A

Caldevilla-Domínguez, D. (2007) Relaciones públicas y cultura. Visión libros.

Caldevilla-Domínguez, D., García García, E. y Barrientos-Báez, A. (2019). La importancia del turismo cultural como medio de dignificación del turista y de la industria. Mediaciones Sociales, 18, 59-69. https://doi.org/10.5209/meso.65117

Caldevilla-Domínguez, D., Barrientos-Báez, A. y Parra-López, E. (2020). Horizontes del mundo digital: de la simulación y la banalización de la experiencia, a un uso social, ecológico e innovador de la Sociedad Red. CIC. Cuadernos de Información y Comunicación, 25, 269-277. https://doi.org/10.5209/ciyc.68722

Caro, J., Luque, A. y Zayas, B. (2014). Aplicaciones tecnológicas para la promoción de los recursos turísticos culturales. XVI Congreso Nacional de Tecnologías de la Información Geográfica. Alicante, España. http://goo.gl/2Svq61

Celso Campos, P. (2010). Jornalismo ambiental, envelhecimento demográfico e universidade aberta à terceira idade: A tese do empoderamento. Perspectivas de la Comunicación, 3(2), 48-63. http://revistas.ufro.cl/ojs/index.php/perspectivas/article/view/95/78 
Caldevilla-Domínguez, D., Barrientos-Báez, A., Pérez-García, A. y Gallego-Jiménez, M. G. El uso de las redes sociales y su relación con la decisión de compra del turista

César Arnaiz, F. y Arnaiz Burne, S. M. (2016). Capitalismo, Sociedad y turismo. Opción, 32(13), 319-338. https://cutt.ly/0jq2wcz

Chen, B. y Marcus, J. (2012). Students' self-presentation on Facebook: An examination of personality and self-construal factors. Computers in Human Behavior, 28(6), 20912099. http://doi.org/10.1016/j.chb.2012.06.013

Chinchay-Villarreyes, S. S., Cango Córdova, J. I., Aldana Tume, A. A. y Seminario Sanz, R. S. (2020). Estrategias de promoción para el fomento del turismo religioso en Perú. Revista de Ciencias Sociales, 26(3), 272-283. https://cutt.ly/6jwouAf

Correyero Ruiz, B. (2003). La propaganda turística española en los años del aislamiento internacional. Historia y Comunicación Social, 8, 47-61. https://revistas.ucm.es/index.php/HICS/article/view/HICS0303110047A

Fernández-Cavia, J., Vinyals-Mirabent, S., Fernández-Planells, A., Weber, W. y Pedraza-Jiménez, R. (2020). Tourist information sources at different stages of the travel experience. El Profesional de la Información, 29(2), 1-12. https://doi.org/10.3145/epi.2020.mar.19

Fiorello, A. (2010). Micro-cluster y nuevo turista. Revista de Comunicación de la SEECI, 23, 1-29. https:// doi.org/10.15198/seeci.2010.23.1-29

Ganga-Contreras, F., Navarrete, E. y Suárez, W. (2017). Aproximación-los fundamentos teóricos de liderazgo auténtico. Revista Venezolana de Gerencia, 22(77), 36-55. https://cutt.ly/cjq2mMW

Gonzálvez Vallés, J. E., Barrientos-Báez, A. y Parra-López, E. (2020). Gobernanza y Comunicación en la Universidad. Estudio-partir del COVID-19. Revista Venezolana de Gerencia, 25(91), 1247-1261. https://cutt.ly/cjwi6nb

Gosling, S., Augustine, A., Vazire, S., Holtzman, N. y Gaddis, S. (2011). Manifestations of Personality in Online Social Networks: Self-Reported FacebookRelated Behaviors and Observable Profile Information. Cyberpsychology, Behavior, and Social Networking, 14(9), 483-488. http://doi.org/10.1089/cyber.2010.0087

Grasmuck, S., Martin, J. y Zhao, S. (2008). Identity construction on Facebook: Digital empowerment in anchored relationships. Computers in Human Behavior, 24(5), 1816-1836. http://doi.org/10.1016/j.chb.2008.02.012

Hays, S., Page, S. y Buhalis, D. (2012). Social Media as a Destination Marketing Tool: Its Use by National Tourism Organizations. Current Issues in Tourism, 16, 1-29. http://doi.org/10.1080/13683500.2012.662215

Infante Limón, E. (2014). Guerra, Historia, turismo y prensa: bases de la puesta en valor del patrimonio cultural de Niebla (Huelva) durante el franquismo. Historia y 
Caldevilla-Domínguez, D., Barrientos-Báez, A., Pérez-García, A. y Gallego-Jiménez, M. G. El uso de las redes sociales y su relación con la decisión de compra del turista

$\begin{array}{lcc}\begin{array}{l}\text { Comunicación } \\ \text { https://doi.org/10.5209/rev_HICS.2014.v19.47290 }\end{array} & \text { 19, 153-170. }\end{array}$

Junco, R. (2011). The relationship between frequency of Facebook use, participation in Facebook activities, and student engagement. Computers and Education, 58(1), 162-171. http:// doi.org/10.1016/j.compedu.2011.08.004

Kosinski, M., Stillwell, D. y Graepel, T. (2013). Private traits and attributes are predictable from digital records of human behavior. Proceedings of the National Academy of Sciences, 110(15), 5802-5805. http://doi.org/10.1073/pnas.1218772110

Lalueza, F. (2019). Tendencias de las redes sociales en 2019: Instagram canibalizará a Facebook. https://cutt.ly/KjqNOCF

Martín-Antoranz, P., Cabezuelo-Lorenzo, F. y Bartolomé-Muñoz-de-Luna, Á. (2019). Competencias y formación ética de los profesionales de la comunicación corporativa en contextos interculturales. Revista de Ciencias de la Comunicación e Información, 24(1), 59-72. https://doi.org/10.35742/rcci.2019.24(1).59-72

Martínez-Valerio, L. (2015). Conocer la personalidad de los universitarios a través de los "me gusta" de Facebook. Prisma Social: revista de ciencias sociales, 15, 147-179.

Matassi, M. y Boczkowski, P. J. (2020). Redes sociales en Iberoamérica. Artículo de revisión. El Profesional de la Información, 29(1), 1-41. https://doi.org/10.3145/epi.2020.ene.04

Mayer, Z. y Puller, S. (2008). The old boy (and girl) network: Social network formation on university campuses. Journal of Public Economics, 92, 329-347. http://doi.org/10.1016/j.jpubeco.2007.09.001

Navarro Celis, F., Almaguer Covarrubias, R., Moreno Velazco, F. y Hernández Rocha, N. P. (2020). Estrategias comunicacionales en el sector turismo. Revista de Ciencias Sociales, 26(1), 77-90. https://cutt.ly/DjwoQG6

Oliveira, A., Correia, A. y Fernandes, C. (2017). A utilização das redes sociais na perspetiva das empresas de turismo em espaço rural. Pasos. Revista de Turismo y Patrimonio Cultural, 15, 807-822. http://doi.org/10.25145/j.pasos.2017.15.055

Ortega, E. (2012). Communication effects at tourism destinations. Proposed model. En A. M. Gil (Coord.), Creating new opportunities in an uncertain environment, XXVI Annual Congress of the European Academy of Management and Business Economics (AEDEM) 1-13. ESIC Editorial.

Parra, P., Gordo, A. J. y D’ Antonio, S. A. (2014). La investigación social aplicada en redes sociales. Una innovación metodológica para el análisis de los «Me gusta» en 
Caldevilla-Domínguez, D., Barrientos-Báez, A., Pérez-García, A. y Gallego-Jiménez, M. G. El uso de las redes sociales y su relación con la decisión de compra del turista

Facebook. Revista Latina de Comunicación Social, 69, 195-212. http://doi.org/10.4185/RLCS-2014-1008

Pempek, T., Yermolayeva, Y. y Calvert, S. (2009). College students' social networking experiences on Facebook. Journal of Applied Developmental Psychology, 30(3), 227-238. http://doi.org/10.1016/j.appdev.2008.12.010

Richards, G. (2004). ¿Nuevos caminos para el turismo cultural?. Association for Tourismand Leisure Educatio (ATLAS).

Salazar Martínez, R. Á., Barrientos-Báez, A. y Caldevilla-Domínguez, D. (2020). Crítica, debate y polarización en torno a la covid-19. Argumentos de Razón Técnica, 23, 151-187. https://doi.org/10.12795/Argumentos/2020.i23.05

Sánchez-Torres, W. C. y Restrepo, J. C. (2015). Marketing en tiempo real y el fortalecimiento del mercadólogo. Escenarios: empresa y territorio, 4(4), 33-52.

Sarmiento Guede, J. S. y Rodríguez Terceño, J. (2018). La comunicación de boca en boca electrónica en los medios sociales. Análisis de sus antecedentes. Revista de Comunicación de la SEECI, 45, 129-145. http://doi.org.10.15198/seeci.2018.45.129$\underline{148}$

Serra, A. (2013). Marketing Turístico. Ediciones Pirámide.

Stutzman, F. (2006). An evaluation of identity-sharing behavior in social network communities. Journal of the International Digital Media and Arts Association, 3(1), 1018.

Suau, F. (2012). El turista 2.0 como receptor de la promoción turística: estrategias lingüísticas e importancia de su estudio. Pasos, Revista de Turismo y Patrimonio Cultural, 10(4), 143-153. https://cutt.ly/pjq9KDK

We are Social. (2019). Global Digital Report. Wearesocial.com. https://wearesocial.com/global-digital-report-2019

\section{AUTOR/ES:}

\section{David Caldevilla-Domínguez:}

Licenciado y Doctor en Ciencias de la Información (Comunicación Audiovisual por la U. Complutense). Diplomado en Magisterio (U. de Zaragoza). Profesor titular en la Facultad de Ciencias de la Información de la UCM. Docente en: U. Complutense, U. Europea de Madrid, IED, ESERP e IPAM (Oporto -Portugal-). Índice h 18. $6^{\circ}$ autor español en trabajos publicados, $13^{\circ}$ en artículos citados y $20^{\circ}$ en citas recibidas de 747 autores totales. Ponente y conferenciante en diversos cursos y profesor en varios títulos propios (Telemadrid, Walter \& Thompson, McCann...). Secretario General de 
Caldevilla-Domínguez, D., Barrientos-Báez, A., Pérez-García, A. y Gallego-Jiménez, M. G. El uso de las redes sociales y su relación con la decisión de compra del turista

la SEECI (Sociedad Española de Estudios de Comunicación Iberoamericana) y del "Fórum Internacional de la Comunicación y Relaciones Públicas" (Fórum XXI). Investigador Principal (IP) del Grupo Complutense de Investigación 'Concilium'. https://orcid.org/0000-0002-9850-1350

\section{Almudena Barrientos-Báez:}

Doctora con mención internacional en Educación y profesora en la Escuela Universitaria de Turismo Iriarte, adscrita a la ULL (Tenerife-España) y en la Univ. Europea y en la UNIR. Máster en Dirección de Protocolo, Producción, Organización y Diseño de Eventos - área de Comunicación - (UCJC) y Máster en Dirección de Alojamientos Turísticos (Univ. Girona). Grado en Turismo (EUTI-ULL) y Magisterio (Univ. Valencia). Forma parte del proyecto DEBATv, Debates Electorales Televisados en España: Modelos, Proceso, Diagnóstico y Propuesta (CSO2017-83159-R), proyecto de I+D+I (Retos) financiado por el Ministerio de Ciencia, Innovación y Universidades y la Agencia Estatal de Investigación (AEI) del Gobierno de España, con el apoyo del Fondo Europeo de Desarrollo Regional (FEDER) de la Unión Europea (UE). Forma parte del proyecto Nuevos valores, gobernanza, financiación y servicios audiovisuales públicos para la sociedad de Internet: contrastes europeos y españoles (RTI2018-096065-B-I00) de investigación del Programa Estatal de I+D+I orientado a los Retos de la Sociedad del Ministerio de Ciencia, Innovación y Universidades (MCIU), Agencia Estatal de Investigación (AEI) y del Fondo Europeo de Desarrollo Regional (FEDER).

https://orcid.org/0000-0001-9913-3353

\section{Álvaro Pérez-García:}

Doctor en Educación por la Universidad de Granada con la tesis titulada: "La integración curricular del cine digital en la formación inicial de los maestros: perspectivas didácticas y creativas". Acreditado como profesor contratado doctor y profesor de universidad privada por la ANECA. Con 1 sexenio de investigación de la CNAI. Actualmente, director del Departamento de Tecnologías de la Información y la Comunicación aplicadas a la Educación, Coordinador Académico de la especialidad de Tecnología e Informática del Máster de Formación del Profesorado de Secundaria y Profesor Adjunto de la Universidad Internacional de la Rioja (UNIR).

Líneas de investigación principales: las TIC aplicadas a la Educación, Cine Educativo, Videojuegos y Educación, e-learning y Didáctica y Organización Escolar.

https://orcid.org/0000-0001-9624-5202

\section{Gloria Gallego-Jiménez:}

Doctora en Educación (Universidad internacional de Catalunya), Máster en Educación Inclusiva (Universidad de Vic). Actualmente profesora de UNIR en Grado de Educación. Actividad investigadora centrada en bullying y educación inclusiva. https:// orcid.org/0000-0003-4498-8869 